\title{
ACHADOS COLONOSCÓPICOS EM PESSOAS SEM QUADRO CLÍNICO DE DOENÇA COLORRETAL
}

\author{
Andy PETROIANU1', Luiz Ronaldo ALBERTI'1 , David Corrêa Alves de LIMA², \\ Heather Lynn HAUTER ${ }^{3}$, Kelly Cristine de Lacerda RODRIGUES ${ }^{3}$ e Julia Cristina de Almeida MENDES ${ }^{4}$
}

\begin{abstract}
RESUMO - Contexto - O câncer colorretal é causa de morte frequente e sua prevenção deve fazer parte dos programas de rastreamento em indivíduos assintomáticos. Objetivo - Avaliar os achados colonoscópicos de pessoas assintomáticas submetidas a colonoscopia. Método - Foram estudados 153 pessoas assintomáticas submetidas a colonoscopia. Sexo, idade, história pregressa e familiar, tabagismo e etilismo foram avaliados. Indivíduos com hematoquesia macro ou microscópica e os com doença colorretal foram excluídos. Preparo intestinal, presença de pólipos, angioectasias, doença diverticular, processo inflamatório e neoplasia foram investigados. Os pólipos foram classificados de acordo com seu tamanho, número e localização. Resultados - A idade média foi de 52,47 $\pm 11,64$ anos. História familiar de câncer colorretal foi verificada em 20,2\% dos indivíduos. Alterações colonoscópicas foram verificadas em 99 indivíduos: pólipos em 64,28\%, doença diverticular em 27,92\%, alterações inflamatórias em 9,74\%, melanose colônica em 2,6\% e angioectasias em 7,8\%. Houve incidência maior de pólipos em indivíduos acima de 50 anos. A análise de regressão logística mostrou a idade e sexo como fatores preditores para a presença de pólipos $(\mathrm{RR}=1,69 ; 1,23<\mathrm{RR}<2,51)$. Conclusão - Existe significativa incidência de alterações colonoscópicas em pessoas assintomáticas submetidas a colonoscopia para rastreamento de câncer colorretal.
\end{abstract}

DESCRITORES - Neoplasias colorretais. Programas de rastreamento. Colonoscopia.

\section{INTRODUÇÃO}

O câncer colorretal (CCR) é uma das neoplasias mais diagnosticadas no mundo. Os programas de rastreamento para essa doença têm grande importância no prognóstico, pois podem detectar lesões précancerosas, como o pólipo adenomatoso, que ao serem retiradas previnem a instalação do câncer ${ }^{(2,3,6 \text {, }}$ ${ }^{22}$. Apesar de ainda não ser possível determinar quais adenomas irão progredir para o câncer, algumas de suas características patológicas estão relacionadas com o risco de progressão para CCR: tamanho maior ou igual a $10 \mathrm{~mm}$, tubuloviloso ou viloso na histologia e displasia moderada ou alta ${ }^{(8,14,18,21)}$. Mesmo quando já houve a transformação maligna, o tratamento do câncer inicial pode curar ou aumentar a sobrevida. Ao contrário de outras doenças em que a forma de rastreamento está bem estabelecida, no CCR há vários métodos em uso. Os exames incluem a pesquisa de sangue oculto nas fezes, a retossigmoidoscopia flexível, a colonoscopia e o enema de duplo contraste ${ }^{(2,3,6,22)}$.

A presença de sangue nas fezes é um exame pouco útil pois são elevados os índices de falso-positivo e de falso-negativo. Mesmo quando positivo, requer outros exames para identificar a origem e causa do sangramento. Nesse sentido, é preferível realizar de início os exames endoscópicos ou de imagem ${ }^{(3,6,22)}$.

A retossigmoidoscopia flexível é limitada, por não permitir o exame da maior parte do cólon. COLLETT et al. ${ }^{(3)}$ encontraram lesões proximais sincrônicas durante colonoscopia em 30\% dos indivíduos com neoplasia distal de cólon, previamente rastreados por sigmoidoscopia flexível. Ao estudarem 2.686 indivíduos assintomáticos com idade igual ou superior a 50 anos, IMPERIALE et al. ${ }^{(6)}$ concluíram que as pessoas com pólipos no cólon distal são mais propensas a desenvolver neoplasia avançada no cólon proximal. Entretanto, se a colonoscopia for realizada apenas nas pessoas com pólipos distais, cerca da metade dos casos de neoplasia avançada em outras partes de cólon não seriam detectadas.

O enema de duplo contraste, apesar de ser sensível mesmo para pequenos pólipos, deixa passar lesões iniciais planas que não provocam defeito de enchimento ${ }^{(3,8)}$. Outro inconveniente é a necessidade de se completar a propedêutica com colonoscopia para biopsiar ou

\footnotetext{
Instituições: Instituto Alfa de Gastroenterologia - Hospital das Clínicas - Universidade Federal de Minas Gerais; Biogastro - Núcleo Avançado de Videoendoscopia Digestiva ${ }^{1}$ Departamento de Cirurgia da Faculdade de Medicina, Universidade Federal de Minas Gerais - UFMG; ${ }^{2}$ Instituto Alfa de Gastroenterologia - Hospital das Clínicas da UFMG; ${ }^{3}$ Médica; ${ }^{4}$ Curso de Medicina da Faculdade de Medicina da UFMG

Correspondência: Prof. Andy Petroianu - Av. Afonso Pena, 1626 - apt. 1901 - 30130-005, Belo Horizonte, MG. E-Mail: petroian@medicina.ufmg.br
} 
retirar completamente a lesão. Sendo assim, é preferível que a colonoscopia seja feita desde o início.

Quanto à colonoscopia, ainda não há estudos aleatórios que mostrem benefício com relação à mortalidade quando esse exame é usado para rastreamento de CCR, mas ela tem sido adotada por permitir a observação de todo o cólon, remoção dos pólipos e outras lesões eventualmente encontradas. O elevado custo em relação aos outros métodos e a possibilidade de complicações durante o procedimento são os principais inconvenientes para seu estabelecimento como exame rotineiro no rastreamento do $\mathrm{CCR}^{(22)}$.

Dentre os fatores de risco para o desenvolvimento de CCR em indivíduos assintomáticos estão a história familiar para parentes de primeiro grau com CCR, tabagismo, alcoolismo moderado ou intenso. Por outro lado, a ingestão habitual de cereais, vitamina D e uso de anti-inflamatórios nãoesteróides (AINEs) reduzem a probabilidade dessa doença. Nenhuma associação foi estabelecida com o índice de massa corporal (IMC), colecistectomia prévia ou nível de colesterol plasmático $^{(9)}$.

A idade que tem sido recomendada para início do rastreamento para câncer colorretal é 50 anos em indivíduos sem história familiar positiva. Nos Estados Unidos, entre $45 \mathrm{e}$ 49 anos, a incidência anual de câncer colorretal é de 24 casos por 100.000 habitantes, enquanto entre 50 e 54 anos, chega a 48 por 100.000 habitantes. Entretanto, aproximadamente 7\% a 30\% dos casos de CCR ocorrem em pessoas abaixo de 50 anos, muitas delas sem fator aparente de risco para a doença ${ }^{(6)}$.

Apesar dos muitos estudos que relacionam o rastreamento do CCR com fatores de risco, ainda há pouca pesquisa na população assintomática no geral. Nesse sentido, o objetivo do presente trabalho foi verificar os achados colonoscópicos em pessoas sem quadro clínico de doença colorretal.

\section{MÉTODO}

Este estudo foi realizado de acordo com as recomendações da Declaração de Helsinque e da Resolução no ${ }^{\circ}$ 196/96 do Ministério da Saúde, sobre pesquisa envolvendo seres humanos $^{(12)}$ e foi aprovada pelo Comitê de Ética em Pesquisa da Universidade Federal de Minas Gerais.

Foram estudados 153 indivíduos consecutivos adultos, de ambos os sexos, assintomáticos, submetidos a colonoscopia, como rastreamento para afecções colorretais na Clínica Biogastro de Belo Horizonte, MG, no período de abril de 2000 a maio de 2006. Trata-se de hospital privado que realiza cerca de 4.000 colonoscopias ao ano. Os indivíduos vieram encaminhados por diversos especialistas (cardiologista, clínico geral, gastroenterologista, ginecologista), que solicitaram o procedimento como exame de prevenção para câncer de cólon.

Todos receberam a mesma orientação para o preparo colônico, com dieta sem resíduos na véspera do exame e dieta líquida exclusiva, além de $10 \mathrm{mg}$ de laxante de contato (bisacodil) no dia anterior à colonoscopia. Foi estimulado o aumento do consumo de líquidos ao longo do preparo, apenas proibindo o consumo de laticínios e bebidas contendo corantes escuros. Quatro horas antes da realização do exame, os indivíduos receberam solução de manitol a $10 \%$, tendo que consumir $1.000 \mathrm{~mL}$ da solução em período de 1 hora.

Os indivíduos foram identificados por sexo e idade, tendo sido pesquisada história pessoal e familiar para carcinomas colorretal, de endométrio, de mama, de bexiga e de rim, além de pólipos colorretais, doença inflamatória intestinal, tabagismo e etilismo. A história familiar foi considerada apenas para irmãos e pais e tios de primeiro grau.

Os exames foram realizados por um único endoscopista em todos os casos. Pessoas com pesquisa de sangue oculto nas fezes positiva, história pessoal de pólipos, CCR ou outra afecção colorretal, dor abdominal crônica ou outros sintomas gastrointestinais, incluindo sangramento, diarreia, alterações recentes de hábito intestinal ou alteração das fezes foram excluídas.

Durante a colonoscopia, foi verificada a qualidade do preparo intestinal, definido como adequado quando foi possível verificar a superfície mucosa desde o reto até o ceco sem a necessidade de lavagens durante o exame e inadequado quando houve a necessidade dessas lavagens. A progressão do aparelho até a transição ileocecal e fundo cecal foi também considerada.

Os seguintes aspectos foram investigados durante o procedimento colonoscópico: presença de pólipos (adenoma tubular, tubuloviloso, viloso, hiperplásicos), angiodisplasias, doença diverticular, inflamação do cólon, ileíte e melanose, e neoplasias. Em todos os casos, foram realizados exames histológicos e caracterização completa da afecção. As neoplasias foram dimensionadas e quantificadas. Em relação aos pólipos, a avaliação macroscópica constituiu-se da caracterização de seu número, tamanho em milímetros e distribuição deles em relação às regiões anatômicas intestinais. Para classificar o padrão das criptas colônicas, com base nos critérios de Kudo, foi feita aspersão do corante de contraste índigo-carmin, seguida de magnificação e cromoendoscopia digital. Os pólipos foram retirados por técnica específica (polipectomia e mucosectomia) e submetidos a estudo histológico.

$\mathrm{O}$ dimensionamento da amostra teve por base o cálculo amostral normal segundo a fórmula: $\mathrm{n}=\mathrm{z}^{2} \alpha_{12} / 4 \mathrm{~d}^{2}$, considerando $\alpha=0,05$ e $\beta=0,90$. Por esse cálculo, a amostra mínima confiável é de 96 indivíduos. Para avaliar a comparabilidade dos grupos com base na idade, realizou-se o teste $t$ de Student para amostras contínuas não pareadas. Para avaliar a homogeneidade da amostra e as diferenças entre os parâmetros, foram utilizados os testes do qui ao quadrado e exato de Fisher. Calculou-se também o odds ratio e o intervalo de confiança das variáveis analisadas. Análise de regressão logística multivariada foi realizada para verificar a relação entre a presença de pólipos e seu fatores preditivos. As diferenças foram consideradas significativas para valores correspondentes a $P<0,05$.

\section{RESULTADOS}

Não houve complicação imediata na presente casuística. Todos os indivíduos recuperaram-se do procedimento anestésico e colonoscópico sem intercorrências. Um paciente 
apresentou sangramento sem repercussão clínica no terceiro dia pós-polipectomia em cólon ascendente, que cessou espontaneamente e requereu apenas suporte clínico, sem necessidade de hemotransfusão, tendo sido internado por 1 dia.

Foram estudados $66(43,1 \%)$ indivíduos do sexo masculino e $87(56,8 \%)$ do sexo feminino. A idade média global foi de $52,47 \pm 11,64$ anos, sendo de 52,03 $\pm 12,37$ entre os homens e de $52,81 \pm 11,13$ entre as mulheres.

O preparo colônico foi adequado em 140 indivíduos $(91,5 \%)$ (58 homens e 82 mulheres), permitindo excelente visão da superfície mucosa do reto ao ceco. Em 14 pessoas $(8$ homens e 6 mulheres) o preparo inicial não foi satisfatório e houve a necessidade de lavagem no período do exame para que houvesse adequada visão da mucosa intestinal. Entretanto, o exame colonoscópico foi possível de ser realizado de forma satisfatória com a progressão do aparelho até o fundo cecal, permitindo observação adequada de todo o intestino grosso, do óstio apendicular e da válvula ileocecal.

História familiar de carcinoma colorretal foi relatada por 31 pessoas (17 homens e 14 mulheres). Nenhum dos 153 pacientes relatou história familiar para carcinoma de endométrio e de bexiga ou rim. História familiar para pólipos colorretais foi identificada em 35 pacientes (13 homens e 22 mulheres). Houve relação entre a história familiar de pólipos e a presença de pólipos nos indivíduos desta casuística $(P=0,023)$. Os demais pacientes $(53$ homens e 66 mulheres) não apresentavam história pessoal ou familiar de pólipos. História familiar de doença inflamatória intestinal foi relatada por apenas quatro pacientes, todas do sexo feminino.

Em relação à neoplasia de outra localização, três homens e duas mulheres relataram história familiar positiva para o carcinoma de mama e quatro mulheres já relataram passado pessoal de câncer de mama.

Tabagismo foi encontrado em 17 indivíduos (13 homens e 4 mulheres). Outros 13 indivíduos ( 8 homens e 5 mulheres) foram tabagistas previamente. Foi observada incidência maior de tabagismo no sexo masculino $(P=0,002$; RR $=4,41 ; 1,51$ $<\mathrm{RR}<12,52)$. Alterações colonoscópicas foram verificadas em 12 indivíduos. Não foi encontrada relação entre o hábito de fumar e a presença de pólipos $(P=0,41)$.

Etilismo esteve presente em 49 pessoas (30 homens e 19 mulheres). Apenas um paciente do sexo masculino relatou ter abandonado o etilismo. A maioria dos indivíduos informou não fazer uso de bebida alcoólica (20 homens e 48 mulheres). Foi observada uma incidência maior de etilismo no sexo masculino $(P=0,0006 ; \mathrm{RR}=2,12 ; 1,33<\mathrm{RR}<3,44)$. Alterações colonoscópicas foram verificadas em 29 indivíduos etilistas. Não foi encontrada relação estatística entre o etilismo e a presença de pólipos colorretais $(P=0,23)$.

Alterações colonoscópicas foram verificadas em 99 indivíduos (64,28\%) (47 homens e 52 mulheres). Não houve diferença na incidência de alterações em relação ao sexo $(P$ $=0,1215 ; \mathrm{RR}=1,19 ; 0,95<\mathrm{RR}<1,50)$. A Tabela 1 descreve as alterações encontras nesses 99 indivíduos. Em 16 pessoas do sexo masculino e 18 do feminino encontraram-se mais de uma alteração colonoscópica.
TABELA 1. Caracterização das alterações colonoscópicas verificadas nos indivíduos da casuística

\begin{tabular}{lccc}
\hline \multirow{2}{*}{ Alterações colonoscópicas } & $\begin{array}{c}\text { \% } \\
\text { (em relação ao total } \\
\text { de indivíduos) }\end{array}$ & Número de indivíduos \\
\cline { 3 - 4 } & 38,5 & 30 & 29 \\
\hline Pólipos & 28,10 & 19 & 24 \\
Doença diverticular & 9,80 & 9 & 6 \\
Reação inflamatória inespecífica & 2,61 & & 4 \\
Melanose & 7,84 & 5 & 7 \\
Alterações vasculares & Mulheres \\
\hline Obs: 16 indivíduos do sexo masculino e 18 do sexo feminino apresentaram mais de uma alteração colonoscópica
\end{tabular}

Pólipos foram verificados em 59 pessoas ( 30 homens e 29 mulheres), correspondendo a 38,3\% desta casuística. Desses, 35 apresentaram apenas um pólipo, 8 tiveram dois pólipos, 3 tiveram quatro pólipos e pólipos múltiplos foram verificados em 12 indivíduos. A Tabela 2 mostra a distribuição de pólipos de acordo com as regiões anatômicas intestinais. Observa-se incidência maior de pólipos múltiplos hiperplásicos na região do reto $(78,57 \%)$ quando comparados a de outras localizações $(P=0,018)$. Observa-se também a elevada incidência de adenomas em região de ceco e cólon ascendente $(45,76 \%)$.

TABELA 2. Distribuição e número de pólipos de acordo com as localizações anatômicas em 59 indivíduos

\begin{tabular}{lcc}
\hline Localização anatômica & $\begin{array}{c}\text { Número de } \\
\text { pólipos }\end{array}$ & $\begin{array}{c}\text { Número de indivíduos com } \\
\text { pólipos múltiplos }\end{array}$ \\
\hline Ceco & 8 & - \\
Cólon ascendente & 19 & 1 \\
Cólon transverso & 15 & 1 \\
Cólon descendente & 7 & 1 \\
Sigmoide & 11 & - \\
Transição retossigmoideana & 2 & $11 *$ \\
Reto & 13 & - \\
\hline$* P=0,018$ & & \\
Obs: 1 . Em dois indivíduos que apresentaram pólipos múltiplos no reto, houve a concomitância de pólipos múltiplos no cólon \\
transverso e cólon ascendente. \\
2.0 número de pólipos na primeira coluna não leva em consideração os pólipos múltiplos
\end{tabular}

Pólipos hiperplásicos foram encontrados em 17,53\% dos indivíduos desse estudo, sendo 11 casos entre os homens e 17 entre as mulheres. Lesões adenocarcinomatosas não foram verificadas. A Tabela 3 mostra a distribuição dos pólipos de acordo com seu tamanho e localização anatômica. Observase que a maioria mediu menos que $10 \mathrm{~mm}$. Entretanto, em dois indivíduos houve pólipos maiores que $20 \mathrm{~mm}$ no cólon descendente e reto.

TABELA 3. Distribuição dos pólipos de acordo com o tamanho em milímetros

\begin{tabular}{lcccc}
\hline Localização anatômica & \multicolumn{4}{c}{ Tamanho dos pólipos } \\
\cline { 2 - 5 } & $<5 \mathrm{~mm}$ & $5-10 \mathrm{~mm}$ & $10-20 \mathrm{~mm}$ & $>20 \mathrm{~mm}$ \\
\hline Ceco & 6 & 2 & - & - \\
Cólon ascendente & 13 & 6 & 1 & - \\
Cólon transverso & 9 & 7 & - & - \\
Cólon descendente & 4 & 3 & - & 1 \\
Sigmóide & 5 & 6 & - & - \\
Transição retossigmoideana & 1 & 1 & - & - \\
Reto & 21 & 2 & - & 1 \\
\hline
\end{tabular}


A presença de adenoma tubular foi verificada em 20,77\% dos indivíduos com alterações colonoscópicas, sendo 22 homens e 10 mulheres $(P=0,003$; RR $=2,90 ; 1,48<\mathrm{RR}<5,70)$. Adenoma tubuloviloso foi verificado em quatro indivíduos (três homens e uma mulher) $(P=0,314)$. Adenoma viloso não foi encontrado nesta série.

Houve incidência maior de pólipos nos indivíduos acima de 50 anos, quando comparada com os de idade inferior $(P=$ $0,018)$. A análise de regressão logística multivariada mostrou serem a idade e o sexo fatores preditores significativos para pólipos ( $\mathrm{RR}=1,69 ; 1,23<\mathrm{RR}<2,51)$.

Doença diverticular foi verificada em $27,92 \%$ do total de indivíduos (19 homens e 24 mulheres). Reação inflamatória inespecífica do cólon foi verificada em $9,74 \%$ dos indivíduos (nove homens e seis mulheres). Ileíte reacional inflamatória não foi encontrada no presente estudo. Melanose colônica foi verificada em 4 indivíduos do sexo feminino e alterações vasculares do tipo angiodisplasia ocorreram em outros 12 indivíduos (Tabela 1).

\section{DISCUSSÃO}

O câncer colorretal, em decorrência da sua prevalência, do longo período assintomático e da existência de lesões pré-cancerosas tratáveis preenche todos os critérios para rastreamento populacional de rotina ${ }^{(13)}$. Dentre os procedimentos utilizados para essa avaliação, a colonoscopia destaca-se pela possibilidade de observação de todo o intestino, além de permitir identificação, diagnóstico correto por meio de biopsias e ressecção das lesões encontradas ${ }^{(10)}$.

A investigação de câncer e afecções pré-cancerosas por meio de colonoscopia pode levar a desconfortos diversos, como ansiedade e incômodos decorrentes do preparo colônico e do próprio exame, principalmente por aspiração incompleta do ar insuflado ${ }^{(1)}$. De acordo com TAUPIN et al. ${ }^{(20)}$, indivíduos submetidos a investigação e tratamento de afeç̧ões, por meio de colonoscopia, melhoram o estado mental e a qualidade de vida. Esse benefício da qualidade de vida pode representar mais um argumento persuasivo para a utilização do rastreamento rotineiro do CCR através de colonoscopia.

De acordo com dados da "American Society for Gastrointestinal Endoscopy", a morbidade do procedimento varia de $0,2 \%$ nas colonoscopias diagnósticas até $1,2 \%$ nas terapêuticas, com mortalidade de $0,0006 \%{ }^{(4)}$. Essa reduzida incidência de complicações, associada à avaliação cardiológica prévia ao procedimento anestesiológico, são responsáveis pela grande aceitação (mais de 94\%) dos indivíduos em se submeterem ao exame ${ }^{(13)}$. Tal dado é frequentemente observado pelos colonoscopistas durante a entrevista que antecede o procedimento colonoscópico. Na presente casuística não foi verificada complicação relacionada ao procedimento colonoscópico.

Foram encontradas lesões pré-malignas em um quarto dos doentes. Essa incidência foi similar à da literatura ${ }^{(13,16)}$. Estudos em necropsias mostram prevalência de $20 \%$ a $30 \%$ para pólipos adenomatosos e referem aumento dessa incidência com a idade ${ }^{(13)}$. O Colégio Americano de Gastroenterologia estabeleceu a incidência média $25 \%$ para adenomas em indivíduos acima de 50 anos e sem história de risco de CCR, percentagem semelhante à detectada no presente estudo ${ }^{(16)}$. Portanto, a retirada dessas lesões por via endoscópica previne o câncer e todas as suas consequências, por vezes fatais.

Verificou-se elevada incidência de adenomas em região de ceco/cólon ascendente. Segundo NEWCOMB et al..$^{(1)}$, em indivíduos com história familiar positiva para neoplasias colorretais, cerca de 30\% a 40\% dos cânceres se desenvolvem no cólon direito. Esses dados mostram a importância da realização completa da colonoscopia até o ceco, como foi feita neste trabalho.

Nesta série, procurou-se identificar fatores preditores para a presença de pólipos. Em publicações prévias, o diagnóstico precoce de câncer em casos índices e o número de parentes afetados foram considerados fatores de risco para a presença de pólipos ${ }^{(7,13)}$. Nessa casuística, verificou-se que a idade precoce de diagnóstico do câncer em familiares aumentou o risco da presença de pólipos em parentes de primeiro grau, quando comparado ao de parentes que apresentaram essa neoplasia após os 62 anos. Além disso, foi verificado que a presença de história familiar de câncer ou pólipo colorretal, independentemente da faixa etária, foi fator preditivo positivo para a presença de pólipos. No geral, houve incidência maior de pólipos após os 50 anos, quando comparada com os indivíduos de idade inferior, conforme encontrado na literatura ${ }^{(7)}$. Considerando a evidência para o efeito protetor da realização de polipectomias durante procedimentos colonoscópicos definida pelo "National Polyp Study" que sugerem diminuição de até $90 \%$ na incidência de $\mathrm{CCR}^{(23)}$, destaca-se a importância do estudo endoscópico mais precoce nos indivíduos com história familiar para essa neoplasia.

Uma limitação da colonoscopia são as lesões despercebidas, mesmo em exames completos até o ceco. REX et al. ${ }^{(15)}$ encontraram taxa média de adenomas despercebidos de $24 \%$ em geral, sendo de $27 \%$ para adenomas menores que $5 \mathrm{~mm}$, $13 \%$ para adenomas entre 6 e $9 \mathrm{~mm}$ e de $6 \%$ para adenomas de pelo menos $10 \mathrm{~mm}$. Tais dados mostram que apesar da colonoscopia ser considerada o padrão-ouro, esse método não é infalível ${ }^{(5)}$.

Diferenças biológicas relacionadas ao sexo podem resultar em diferentes expressões fenotípicas de CCR entre homens e mulheres. A prevalência corrigida pela idade de adenomas e CCR é maior entre os homens do que as mulheres ${ }^{(6,22)}$. De fato, no presente estudo, encontrou-se prevalência maior de adenomas no sexo masculino (OR 3,70). No entanto, a prevalência geral de alterações colonoscópicas não variou entre os sexos, justificando a necessidade de realização de colonoscopias também entre as mulheres. Esses dados foram também confirmados por SAINI et al. ${ }^{(17)}$ que estudaram 1.463 mulheres e detectaram adenomas em $20,4 \%$ e neoplasia avançada em $4,9 \%$ dos casos.

Foi possível a intubação do ceco em todas as colonoscopias. Esse resultado está de acordo com os estudos americanos que relatam a taxa de intubação de $97 \% \%^{(15,19)}$. Tal fato possivelmente foi facilitado pela realização de sedação consciente, que permitiu o emprego de manobras colonoscópicas para atingir-se 
o ceco. Não foi verificada complicação relacionada ao ato anestésico e os exames foram realizados sem intercorrências. Esses resultados satisfatórios foram em parte devido à consulta prévia ao procedimento colonoscópico que pode identificar fatores de risco para a anestesia e tratá-los de forma eficaz. De fato, durante a consulta prévia, foram verificados em um paciente fatores de risco mais evidentes para complicações e alterações eletrocardiográficas, que motivaram seu encaminhamento para estudo cardiológico mais pormenorizado, que contraindicou o procedimento colonoscópico num primeiro momento. Esse paciente foi submetido a cirurgia de revascularização miocárdica e após cerca de 6 meses, realizou-se então a colonoscopia.

A extrapolação dos dados deste trabalho deve ser feita com cautela, já que a maioria dos indivíduos desta casuística possuía alto nível sociocultural, além de terem sido esclarecidos quanto à necessidade de colonoscopias para o rastreamento de câncer colorretal. Por estes motivos, é possível encontrar maior incidência de pólipos com displasia de alto grau/carcinoma in situ e até mesmo carcinoma colorretal em indivíduos com menos acesso aos recursos de saúde e informação, já que os mesmos não são submetidos a rastreamento para essa neoplasia e a colonoscopia ocorre na presença de manifestações clínicas de doença.

\section{CONCLUSÃO}

A realização de colonoscopias em pessoas assintomáticas para rastreamento do CCR detecta alterações em cerca de $65 \%$ delas. A possibilidade de tratamento endoscópico das alterações encontradas, especialmente os pólipos, pode levar à diminuição da incidência de CCR.

Petroianu A, Alberti LR, Lima DCA, Hauter HL, Rodrigues KCL, Mendes JCA. Colonoscopic findings in asymptomatic people. Arq Gastroenterol. 2009;46(3):173-8.

ABSTRACT - Context - Colorectal cancer is the second leading cause of cancer-related death. Prevention of colorectal cancer should be achievable by screening programs in asymptomatic patients. Objective - To assess the colonoscopic findings in asymptomatic people submitted to screening. Methods - A prospective study was undertaken on 153 consecutive asymptomatic people submitted to colonoscopy. Sex, age, previous diseases and familial cases of cancer, as well as tobacco and alcohol ingestion were assessed. Patients with rectal macro- or microscopic bleeding and colorectal diseases were excluded. Bowel preparation, polyps, angioectasias, diverticular disease, inflammation and neoplasm were also verified. Polyps were classified according to their size, number and location. Results - Mean age was $52.5 \pm 11.7$ years. Family history for colorectal cancer occurred in $79.8 \%$ of individuals. Colonoscopic alterations were detected in 99 individuals: polyps in $64.3 \%$, diverticular disease in $27.9 \%$, inflammatory mucosal alterations in $9.7 \%$, melanosi coli in $2.6 \%$ and angioectasias in $7.8 \%$. There were an increasing incidence of polyps in patients older than 50 year. Multivariate logistic regression showed age and sex as predictive factors for polyps ( $\mathrm{OR}=1.43 ; 1.19<\mathrm{OR}<2.67)$. Conclusion - There is a significant incidence of colonoscopic alterations in asymptomatic people submitted to colonoscopy for colorectal cancer screening.

HEADINGS - Colorectal neoplasms. Mass screening. Colonoscopy. 


\section{REFERÊNCIAS}

1. Brett J, Bankhead C, Henderson B, Watson E, Austoker J. The psychological impact of mammographic screening: a systematic-review. Psychooncology. 2005; 14:917-38.

2. Capobiango A, Araujo ID, Petroianu A. Estudo epidemiológico das neoplasias malignas do intestino grosso e ânus no estado de Minas Gerais. Rev Bras Coloproctol. 1992;12:5-8

3. Collett JA, Platell C, Fletcher DR, Aquilia S, Olynyk JK. Distal colonic neoplasms predict proximal neoplasia in average risk asymptomatic subjects. J Gastroenterol Hepatol. 1999;14:67-71.

4. Dominitz JA, Eisen GM, Baron TH, Goldstein JL, Hirota WK, Jacobson BC, Johanson JF, Leighton JA, Mallery JS, Raddawi HM, Vargo JJ 2nd, Waring JP, Fanelli RD, Wheeler-Harbough J, Faigel DO; Standards of Practice Committee. American Society for Gastrointestinal Endoscopy. Complications of colonoscopy. Gastrointest Endosc. 2003;57:441-5.

5. Huang CS, Lal SK, Farraye FA. Colorectal cancer screening in average risk individuals. Cancer Causes Control. 2005;16:171-88.

6. Imperiale TF, Wagner DR, Lin CY, Larkin GN, Rogge JD, Ransohoff DF. Risk of advanced proximal neoplasms in asymptomatic adults according to the distal colorectal findings. N Engl J Med. 2000;343:169-74.

7. Johns LE, Houlston RS. A systematic review and meta-analysis of familiar colorectal cancer risk. Am J Gastroenterol. 2001;96:2992-3.

8. Levin TR, Palitz A, Grossman S, Conell C, Finkler L, Ackerson L, Rumore G, Selby JV. Predicting advanced proximal colonic neoplasia with screening sigmoidoscopy. JAMA. 1999;281:1611-7.

9. Lieberman DA, Prindiville S, Weiss DG, Willett W; VA Cooperative Study Group 380. Risk factors for advanced colonic neoplasia and hyperplastic polyps in asymptomatic individuals. JAMA. 2003;290:2959-67.

10. Lin OS, Kozarek RA, Schembre DB, Ayub K, Gluck M, Cantone N, Soon MS, Dominitz JA. Risk stratification for colon neoplasia: screening strategies using colonoscopy and computerized tomographic colonography. Gastroenterology. 2006;131:1011-9.

11. Newcomb PA, Taylor JO, Trentham-Dietz. Interation of a familial and hormonal risk factors for large bowel cancer in women. Int J Epidemiol. 1999;28:603-8.

12. Petroianu A. Pesquisa em medicina. In: Petroianu A. Ética, moral e deontologia médicas. Rio de Janeiro: Guanabara Koogan; 2000. p.174-8.

13. Pezzoli A, Matarese V, Rubini M, Simoni M, Caravelli GC, Stockbrugger R, Cifala V, Boccia S, Feo C, Simone L, Trevisani L, Liboni A, Gullini S. Colorectal cancer screening: results of a 5-year program in asymptomatic subjects at increased risk. Dig Liver Dis. 2007;39:33-9.

14. Read TE, Read JD, Butterly LF. Importance of adenomas $5 \mathrm{~mm}$ or less in diameter that are detected by sigmoidoscopy. N Engl J Med. 1997;336:8-12.

15. Rex DK, Cutler CS, Lemmel GT, Rahmani EY, Clark DW, Helper DJ, Lehman GA, Mark DG. Colonoscopic miss rates of adenomas determined by back-toback colonoscopies. Gastroenterology. 1997;12:24-8.

16. Rex DK, Petrini JL, Baron TH, Chak A, Cohen J, Deal SE, Hoffman B, Jacobson BC, Mergener K, Petersen BT, Safdi MA, Faigel DO, Pike IM; ASGE/ACG Taskforce on Quality in Endoscopy. Quality indicators for colonoscopy. Am J Gastroenterol. 2006;101:873-85.

17. Saini SD, Kim HM, Schoenfeld P. Incidence of advanced adenomas at surveillance colonoscopy in patients with a personal history of colon adenomas: a meta-analysis and systematic review. Gastrointest Endosc. 2006;64:614-26.

18. Schoen RE, Corle D, Cranston L, Weissfeld JL, Lance P, Burt R, Iber F, Shike M, Kikendall JW, Hasson M, Lewin KJ, Appelman HD, Paskett E, Selby JV, Lanza $\mathrm{E}$, Schatzkin A. Is colonoscopy needed for the nonadvanced adenoma found on sigmoidoscopy? The Polyp Prevention Trial. Gastroenterology. 1998;115:533-41.

19. Schoenfeld P, Cash B, Flood A, Dobhan R, Eastone J, Coyle W, Kikendall JW, Kim HM, Weiss DG, Emory T, Schatzkin A, Lieberman D; CONCeRN Study Investigators. Colonoscopic screening of average-risk women for colorectal neoplasia. N Engl J Med. 2005;352:2061-8.

20. Taupin D, Chambers SL, Corbett M, Shadbolt B. Colonoscopic screening for colorectal cancer improves quality of life measures: a population-based screening study. Health Qual Life Outcomes 2006;4:82-9.

21. Wallace MB, Kemp JA, Trnka YM, Donovan JM, Farraye FA. Is colonoscopy indicated for small adenomas found by screening flexible sigmoidoscopy? Ann Intern Med. 1998;129:273-8.

22. Winawer S, Fletcher R, Rex D, Bond J, Burt R, Ferrucci J, Ganiats T, Levin T, Woolf S, Johnson D, Kirk L, Litin S, Simmang C; Gastrointestinal Consortium Panel. Colorectal cancer screening and surveillance: clinical guidelines and rationale-update based on new evidence. Gastroenterology. 2003;124:544-60.

23. Winawer SJ; NPS investigators. The achievements, impact, and future of the National Polyp Study. Gastrointest Endosc. 2006;64:975-8.

Recebido em 23/6/2008 Aprovado em 7/1/2009. 